\title{
Dynamic Consideration of Crowded Magnetic Particle Behavior in Magnetic Field Assisted Finishing Process
}

\author{
Masayuki NATSUME $^{1}$, Takeo SHINMURA ${ }^{2}$ and Hitomi YAMAGUCHI ${ }^{3}$ \\ ${ }^{1}$ Mechanical Systems Department, Nagoya Municipal Industrial Research Institute, Japan, natsume@nmiri.city.nagoya.jp \\ ${ }^{2}$ Graduate School of Engineering, Utsunomiya University, Japan, shinmura@cc.utsunomiya-u.ac.jp \\ ${ }^{3}$ Faculty of Engineering, Utsunomiya University, Japan, hitomiy@cc.utsunomiya-u.ac.jp
}

\begin{abstract}
:
Magnetic force acting on each particle based on magnetic field data is analyzed in this report, by thinking of structure of brush-like cohering magnetic particles as a machining tool of magnetic assisted finishing process. The resultant explanation is that horizontal magnetic force in centripetal direction introduces downward force to workpiece surface in upward magnetic field, and that the force transmission formation made of crowded magnetic particles generates strong pressing force when machining resistance affects the move of magnetic particles on the occasion of machining. Knowledge on difference of magnetic particle behavior, whether resistant force transmission mode occurs or not, will materialize to select hard removing or fine finishing.
\end{abstract}

Keywords: Magnetic field assisted finishing process, Magnetic abrasive, Lubricant, Magnetic force

\section{Introduction}

Application of magnetic materials held in magnetic field can realize surface finishing or deburring, generally cooperated with micro cutting behavior of abrasives mixed. The finishing is performed with the effect of machining force generated by magnetic particles or magnetic tools in magnetic field, so that the force is regarded as an important factor. Resultant machining force of magnetic effect has been considered to be fixed by intensity and gradient of magnetic field as well as physical characteristics of materials in use. However, some different results were observed in experiments [1] [2], which included remarkable change of measured force in normal direction to workpiece surface in constant magnetic field during finishing process. This phenomena show the fact that estimation of magnetic force in static state does not provide enough information for discussion of machining characteristics.

In previous researches, some explanations have been presented on magnetic force which contribute magnetic assisted machining; for example, a report by Shinmura et al.[3], or that by Mori et al.[4] can be found. However, the force of magnetic particle in static state and that during machining operation are treated as same value in all of the reports, except for the report of experimental results of using vertical vibration by Yin et al.[5] Therefore, the research is hard to find that achieves comparative study of the force transmission of magnetic particles in static and machining state.

This report presents dynamic explanation by the examination of the magnetic field structure and by the calculation of magnetic force acting on the particle, on the assumption that the pressing force changes by other factors than magnetic conditions.

\section{Vertical force generated by horizontal gradient of magnetic field}

Plane magnetic abrasive finishing process is executed with magnetic particles held by magnetic field around magnetic pole placed in vertical direction. An experimental equipment concerned is shown in Fig. 1. Magnetic field displayed by magnetic flux density vector is shown in Fig. 2.

In this magnetic field, vertical force acting on magnetic particle looks upward, and does not contribute to press force on the workpiece surface. Existing reports cannot explain this contradiction of difference between magnetic field direction and force direction, because they evaluated normal force, parallel to vertical direction, without detail calculation of magnetic field. Then, this report explain the magnetic force as follows.

Assuming that the size of magnetic particle is so small as to ignore the intensity inclination of magnetic

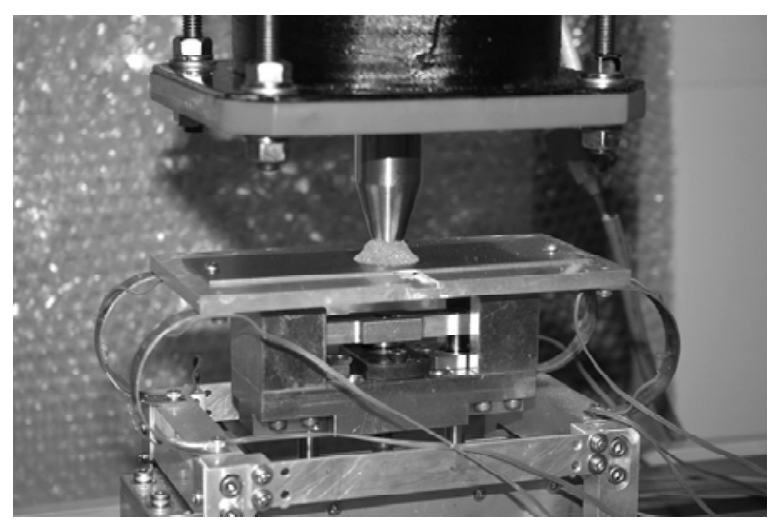

Fig. 1 Machining equipment for consideration 


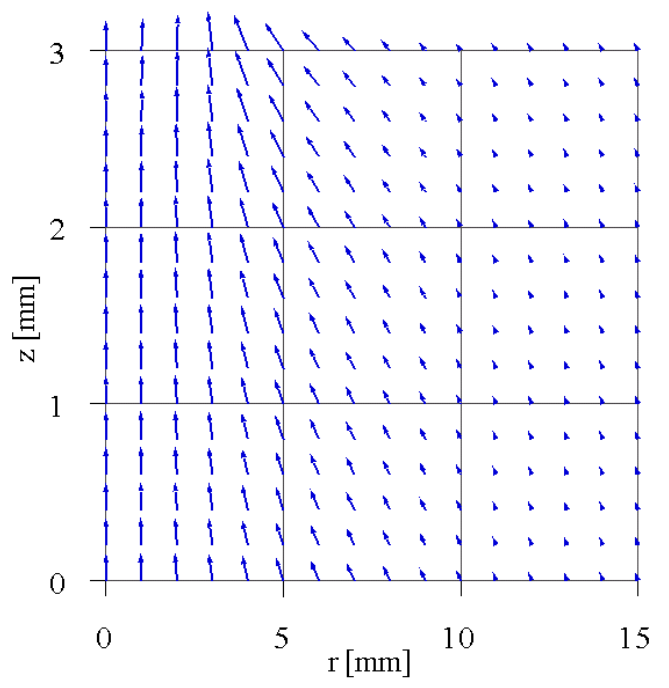

Fig. 2 Magnetic field in machining area

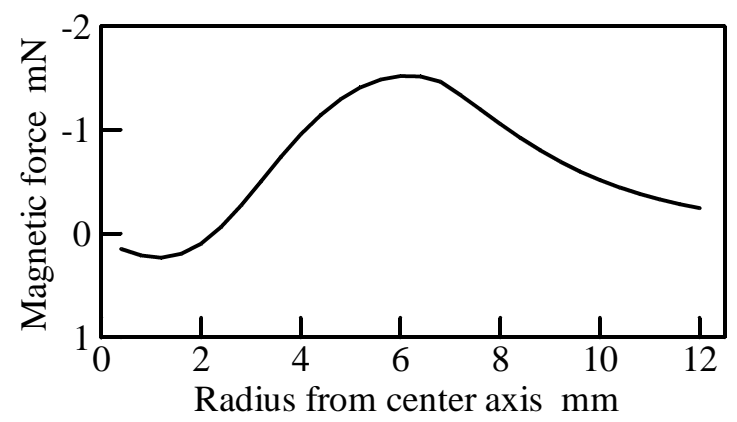

Fig. 3 Magnetic force in inner direction on $1 \mathrm{~mm}$ -height plane

(Negative quantity means inner direction.)

field around the particle, the force acting on a magnetic substance in magnetic field $\boldsymbol{H}$ is described as follows;

$$
\boldsymbol{F}=\operatorname{grad}(\boldsymbol{m} \cdot \boldsymbol{H})
$$

The $\boldsymbol{m}$ is magnetic moment, expressed as follows;

$$
\begin{aligned}
& m \\
& =\int_{v} P_{m} d v \\
& =V\left(1+\chi_{v}\right) \mu_{0} H
\end{aligned}
$$

where $V$ : volume of magnetic substance, $\chi_{v}$ : relative susceptibility of magnetic substance, $\mu_{0}$ : permeability of vacuum.

When $\chi_{v}>>1$, magnetic moment can be written as follows;

$$
\boldsymbol{m} \fallingdotseq V \chi_{v} \mu_{0} \boldsymbol{H}
$$

Therefore, magnetic force in $\mathrm{x}$-axis is written as follows,

$$
\begin{aligned}
& F_{x} \\
& =\partial\left(V x_{v} \mu_{0} H^{2}\right) / \partial x \\
& =2 V \quad x_{v} \mu_{0} H(\partial H / \partial x)
\end{aligned}
$$

$$
=2 V x_{v}\left(B / \mu_{0}\right)(\partial B / \partial x)
$$

where $B$ : magnetic flux density, and $B=\mu_{0} H$.

Concerning demagnetizing field, the force $F_{x}$ is;

$$
F_{x}=2 V\left\{x_{v} /\left(1+x_{v} D\right)\right\}\left(B / \mu_{0}\right)(\partial B / \partial x)
$$

where $D$ : demagnetizing factor.

When one magnetic particle is situated in the magnetic field shown in Fig. 2, the force acting on the particle is given as Eq. (5). Magnetic force distribution at each point calculated by the equation is shown in Fig. 3 , where the height is $1 \mathrm{~mm}$ from workpiece surface, in the condition of $0.4 \mathrm{~mm}$ as sphere diameter of magnetic particle, 1000 as relative susceptibility of magnetic substance and 0.3 as demagnetizing factor. Magnetic field applied to the calculation is decided on the condition that magnetic charge is distributed around the tip of magnetic pole, and is compensated to measured value. Figure 3 displays centripetal force being generated in main part except for a little part near the center axis.

The summation of calculated centripetal force of all the particles on $1 \mathrm{~mm}$-height plane is $3 \mathrm{~N}$, and summation of vertical force of them is $0.8 \mathrm{~N}$.

Coulomb's force acts on neighboring particle each other in crowded distribution of magnetic abrasives, because magnetic charge appears at both ends. Nevertheless, in the case that contact of $\mathrm{N}$-pole and S-pole causes a decrease of surface magnetic charge, the effect of Coulomb's force can be ignored in the consideration, although the magnetic charge does not enough disappear at all like as continuous substance. At least, the magnetic force is qualitatively corresponding to the tendency of real force.

Magnetic particle at the end of magnetic brush is powered by the resultant force of the effect of magnetic field and other magnetic charge, which is look upward; if the resultant magnetic force acted as pressure to workpiece downward, magnetic particle would be left on the surface of workpiece when magnetic pole were pulled up.

Finally, we conclude that measured press force to workpiece surface is generated by transformation of centripetal force. When vertical force were all, normal force to the workpiece would be constant whether in static or in process.

\section{Force transmission formation in magnetic particle brush}

Measured press force, for example, got by the equipment as Fig. 1 is shown in Fig. 4. After starting machining, normal press force increases dramatically to the value of almost two times larger than that in static. The increase ratio of normal force has the character that the ratio is depend on friction coefficient of lubricant supplied [6]. This phenomenon can not be explained with the mechanism of press force generation mentioned above. 
Here, we discuss the inner pressure increase in magnetic particle brush caused by centripetal force. Magnetic particles cohere in the center area of particle brush, and some part of centripetal force generate normal pressure in static state. When cutting resistance is added to the state, particle formation will change.

Surmised schematic of particle formation is shown in Fig. 5. Left-hand figure (a) displays a situation of a few particles lined with large interval, when the line of particle will have few contact to other lines and only the force oriented by magnetic condition acts on workpiece.

Right-hand figure (b) shows cohering particles. Particle lines are inclined by horizontal force at the tip of lines in condition of process. In this condition, cutting resistance, which acts on particles at the tip of lines, is regarded to transmitted by cohering particles. The primary particle lines clear the resistance by inclining, and secondary formation with particles in neighbor lines endure resistant force. The structure made of cohering particles can grow rigid like as rock wall.

For continuing circular motion of the particle structure, even if some particle might be replaced, centripetal force requires enough intensity to keep coherence.

Let the value of centripetal force assume to equal the force which turn transmitted force at the contact point of particles to the direction being at angle as large as center angle of own diameter, shown in Fig. 6.

When the increased value of measured normal force, $F \mathrm{~N}$, in machining process is sheared by $n$ particles, each particle on one plane is required to transmit $F / n$ $\mathrm{mN}$ force in vertical direction. Applying generally used ratio of normal component of cutting resistance to tangential component $\mathrm{c}=F_{v} / F_{h}=2$ to this estimation, horizontal component of required force $F_{h}$ is $F /(2 n)$ $\mathrm{mN}$.

Then, corresponding centripetal force $F_{i}$ to keep the circular motion is written as follows;

$$
F_{i}=F_{h} \cdot \sin \left(d / 2 r_{i}\right)
$$

where $d_{m}$ : diameter of magnetic particle, $r_{i}$ : radius at the position of particle $i$. Sammation of the force of all particles on the circle of radius $r_{i}$ is given as follows;

$$
\begin{aligned}
& \sum F_{i} \\
& =F_{h} \cdot \sin \left(d_{m} / 2 r_{i}\right)\left(2 \pi r_{i} / d_{m}\right) \\
& =\pi F_{h} \cdot\left\{\sin \left(d_{m} / 2 r_{i}\right) /\left(d_{m} / 2 r_{i}\right)\right\}
\end{aligned}
$$

When radius $r_{i}$ becomes larger, $d_{m} / 2 r_{i}$ decreases to 0 , and $\sin \left(d_{m} / 2 r_{i}\right) /\left(d_{m} / 2 r_{i}\right)$ reaches to 1 . Therefore, Eq. (7) changes to following equation corresponding only to $F_{h}$.

$$
\sum F_{i}=\pi F_{h}
$$

When magnetic particles make cohering circles from

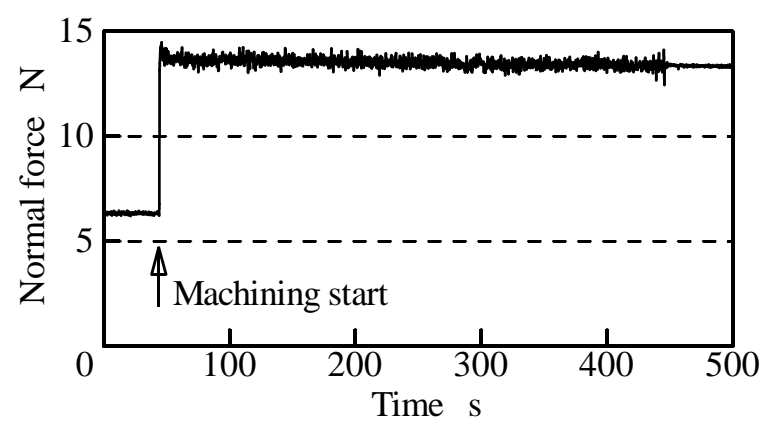

Fig. 4 Change of normal force of magnetic brush [Conditions] Pole revolution: 600 $\mathrm{min}^{-1}$, exciting current: 3A, working clearance: 3mm, amount of magnetic particle: 5g, abrasive: WA\#1500

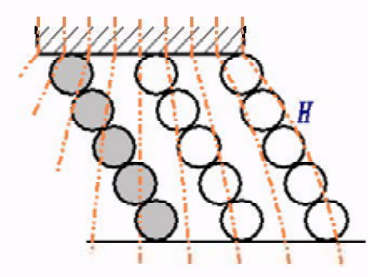

(a)

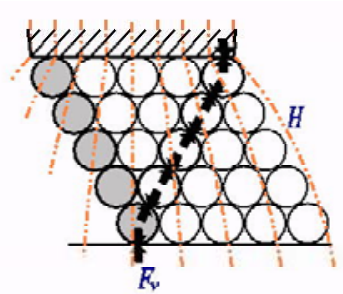

(b)
Fig. 5 Surmised schematic of particle formation

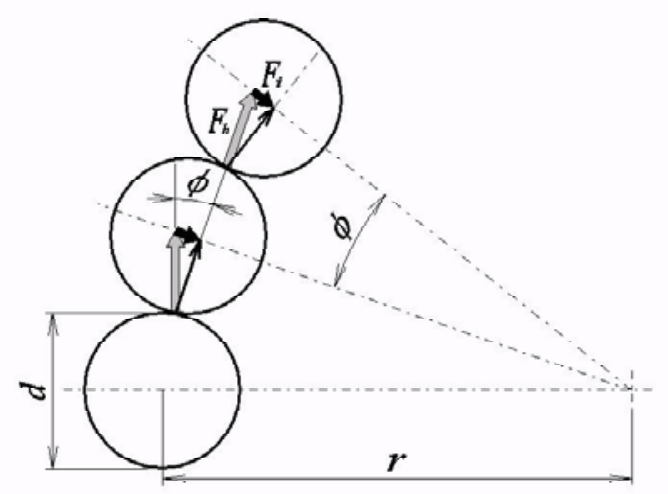

Fig. 6 Schematic of centripetal force and circular particle line

the center to the radius as large as the outline of magnetic pole, the number of particle-lined circles is given as $d_{p} / 2 d_{m}$, where $d_{p}$ : outer diameter of magnetic pole. Then, all the centripetal force to keep the circulate motion of particles in the area is written as follows;

$$
\sum \Sigma F_{i}=\pi d_{p} F_{h} / 2 d_{m}
$$

Substituting values at $1 \mathrm{~mm}$-height plane into Eq. (9), centripetal force required is $0.21 \mathrm{~N}$ on the conditions of $d_{p}=10 \mathrm{~mm}$ as the outer diameter of magnetic pole, $d_{m}=0.4 \mathrm{~mm}$ as the outer diameter of magnetic pole, $n=640$ as the number of magnetic particles, and $F=7 \mathrm{~N}$ as the increase of normal press force. Of course, centrifugal force acts on every particles; it is no more than $0.03 \mathrm{~N}$ estimated for 640 
particles, when revolution number is $600 \mathrm{~min}^{-1}$. Then, it is clarified that centripetal force $2.98 \mathrm{~N}$ generated by circumscribed particles has enough intensity for keeping the circulate motion of core particles.

On the other hand, vertical component of transmitted force dose not require the circulate motion. Although the vertical transmission structure might break down like as buckling, it can also be kept by centripetal force of circumscribed particles.

Finally, it is confirmed that the force transmission structure made of cohering particles props between magnetic pole and workpiece, and causes press force increase originated by reaction force against cutting resistance. This result suggests that the friction of contact point affects the intensity of transmitted force.

If the increase of normal force is needed to be controlled, the transmission structure should be broken by setting such condition that, for example, high horizontal speed makes the particle line inclination larger than stable angle for keeping secondary particle lines. This technique has been proposed by Anzai et al. [7], and this report explain the mechanism.

\section{Conclusions}

As to the phenomena of press force change depending on out of magnetic conditions, magnetic force acting on magnetic particles was calculated by analyzing magnetic field, and dynamic explanation was achieved. We proposed a new view that inner pressure increase of magnetic particle brush oriented by radius magnetic force generated press force in normal direction. And we showed the idea that magnetic particle held in the center area transmitted cutting resistance, and we explained the mechanism of press force increase being larger than that of static state. It was considered in the case of the machining setup that the end surface of magnetic pole looks to workpiece with short clearance, e.g. plane magnetic finishing.

We hope this result will produce new application using magnetic characteristics of particles.

\section{Acknowledgments}

The authors wish to thank Mr.Sei-ichi Matsushita and Dr.Kiwamu Oda of Nagoya Municipal Industrial Research Institute for their technical supports. The authors are thankful to Powdertech Co., Ltd. for supplying iron powder.

\section{References}

[1] Natsume, M., Shinmura, T. and Yamaguchi, H., 2003, Characteristics of Normal Force Generated by Magnetic Particle Blush with Abrasive Slurry, Proc. Autumn meeting of Japan Soc. of Prec. Engg., p210 ( in Japanese ) .

[2] Kawakubo, H., Tsuchiya, K. and Shinmura, T., 2003, Consideration on Mechanics of Polishing Force in Magnetic Polishing Method using Loose Abrasives, J. of the Japan Soc. for Abrasive Technology, Vol.47,
No.12, pp.672-676 ( in Japanese) .

[3] Shinmura, T., Wang, F. and Aizawa, T., 1993, Study on a New Finishing Process of Fine Ceramics by Magnetic Abrasive Machining, J. of Japan Soc. of Prec. Engg., Vol.59, No.8, pp.1251-1256 ( in Japanese) .

[4] Mori, T., Hirota, K., Senda, S. and Kawashima, Y., 2002, Research on Mechanics of Magnetic Abrasive Finishing, Trans. Japan Soc. Mech. Eng., Series C, Vol. 68, No.671 , pp. 2157-2162 (in Japanese) .

[5] Yin, S. and Shinmura, T., 2002, Study of Vibration Assisted Magnetic Abrasive Finishing Process (2nd Report), Trans. Japan Soc. Mech. Eng., Series C, Vol. 68, No.672, pp. 2464-2471 (in Japanese).

[6] Natsume, M., Shinmura, T. and Yamaguchi, H., 2003, The Effects of Lubricant on the Characteristics of the Magnetic Abrasive Brush in Magnetic Abrasive Finishing, Proc. of 2nd International Conf. on LEM21, pp.397-402.

[7] Anzai, M., Kawashima, E., Otaki, H. and Nakagawa, T., 1992, Occurrence Example and Prevention of Scratch in Magnetic Assisted Polishing (in Japanese), Proceedings of 1992 Autumn Meeting of the Japan Society for Precison Engineering, p.127. 